\title{
Initial Operation of the NanoMAX Beamline at MAX IV
}

U. Johansson $^{1 *}$, D. Carbone ${ }^{1}$, S. Kalbfleisch ${ }^{1}$, A. Björling ${ }^{1}$, A. Rodriguez-Frenandez ${ }^{1}$, T. Stankevic ${ }^{1}$, M. Liebi $^{1}$, B. Bring ${ }^{1}$, A. Mikkelsen ${ }^{2}$ and U. Vogt ${ }^{3}$

1. MAX IV Laboratory, Lund University, Lund, Sweden

2. Synchrotron Radiation Research, Department of Physics, Lund University, Lund, Sweden

3. Biomedical \& X-Ray Physics, Dept. of Appl. Physics, KTH Royal Inst. of Tech., Stockholm, Sweden

* Corresponding author, ulf.johansson@maxiv.lu.se

NanoMAX is a hard X-ray nanoprobe beamline at the $3 \mathrm{GeV}$ multi-bend achromat storage ring MAX IV, Lund, Sweden [1]. The beamline is designed to utilize the uniquely high brilliance of the facility to achieve nanometer sized coherent foci with high photon intensity. The small focus is used for scanning imaging with methods such as nano-diffraction, phase and absorption contrast, coherent diffractive imaging and ptychography - forward and Bragg condition. The beamline optics have been briefly presented earlier [2].

The beamline will have two experimental stations when the buildup phase ends 2019/2020. One experimental station, using Kirkpatrick-Baez mirror optics (KB) for focusing, is in limited operation since April 2017 and functionality is added sequentially (Fig 1). The KB system gives a diffraction limited probe of $40 \mathrm{~nm}(24 \mathrm{keV})-200 \mathrm{~nm}(5 \mathrm{keV})$ with $100 \mathrm{~mm}$ working distance from optics to sample position [3]. The generous space will allow for versatile, user defined, sample environments. Three main detectors are planned for or installed at the station; a megapixel photon counting area detector in forward direction, a compact photon counting area detector in off-axis position on a commercial industry robot and a 3element Germanium X-ray fluorescence detector. A compact two axis high precision goniometer will allow advanced studies of ordered samples with diffractive methods. Continuous sample scanning is implemented in a basic version and will be further developed for efficient data acquisition. The design of the second experimental station is ongoing and first tests are anticipated early 2019. The station will use Fresnel zone plates (FZP:s) developed at KTH in Sweden [4, 5] to achieve direct spatial resolution of 30$100 \mathrm{~nm}$ in the energy range 5-12 keV, for 2D and 3D tomographic imaging with X-ray fluorescence, ptychograhy and nano diffraction contrast. In a mature state of the FZP station, the aim is to offer cryocooling and automated sample transfer with improved optics, reaching $10 \mathrm{~nm}$ direct resolution. Both stations are designed in-house with the MAX IV engineering team.

NanoMAX is one of three beamlines at MAX IV to be in early operation, together with BioMAX (Molecular X-ray diffraction) and Hippie (High pressure X-ray photoelectron spectroscopy). We have provided beamtime to a handful user experiments during the first year of operation. Experiments in X-ray fluorescence, wide-angle scattering, nano-diffraction and ptychography have been executed. To achieve optimal performance from the super-polished KB-mirrors we have developed a simple procedure to measure focus astigmatism by scanning a Siemens star like test structure (Fig 2) in ptychographic mode. The test sample image and the probe are reconstructed at the sample position using diffraction data from an inline pixel detector [6]. The probe is then propagated along the beam direction to show the beam profile in the vertical and horizontal planes, as seen at bottom of Figure 3. From the plots, the degree of astigmatism is deduced, the pitch angle adjustments of one or both mirrors are calculated and applied. Most often the optimal focus is achieved in 1-2 iterations. The complete procedure of measuring, reconstructing, propagating, adjusting and verifying normally takes less than 30 minutes. Our experience is that the adjustment stays optimized for several days unless large interventions are made in the 
experimental hutch.

The beamline is, as of spring 2018, in the design phase, commissioning phase and in early user operation simultaneously [7]. The KB station is expected to provide its main capabilities by the end of 2018. For the FZP station the goal is to welcome first users in late 2019 [8].

[1] P F Tavares et al, J. Synchrotron Rad., 21 (2014), p. 862.

[2] U Johansson, U Vogt and A Mikkelsen, Proc. SPIE, 8851 (2013), p. 0L.

[3] K Yamauchi et al, Rev. Sci. Instrum., 73 (2002), p. 4028.

[4] K Parfeniukas et al, Microelectron. Eng. 152 (2016) p. 6.

[5] R Akan et al, RCS Adv. 8 (2018), p. 12628.

[6] B Enders and P Thibault, Proc. R. Soc. A, (2016) 472, p. 0640

[7] U Vogt et al, Proc. SPIE, 10389 (2017), p. 0K.

[8] The authors acknowledge funding of the NanoMAX beamline by the Knut \& Alice Wallenberg Foundation and twelve Swedish universities.

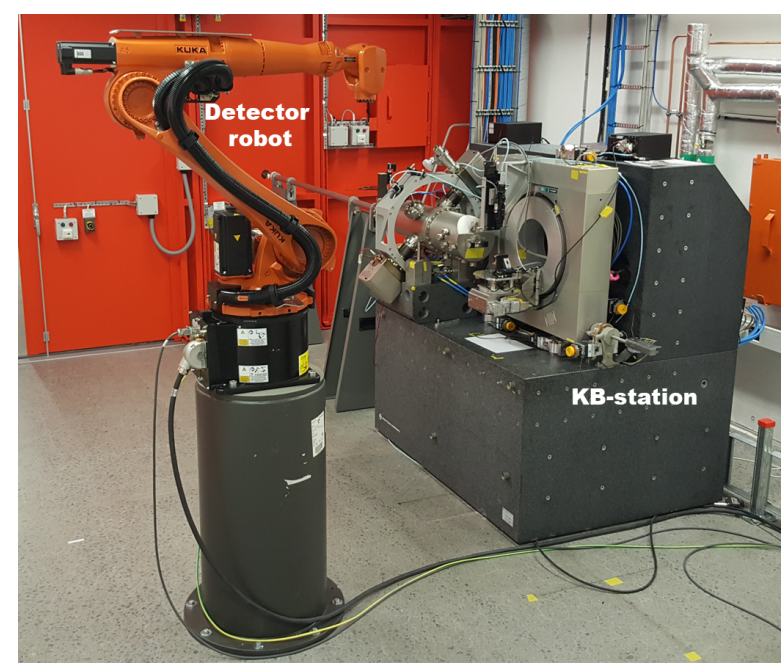

Figure. 1. Photo of the KB experimental station with the detector robot in front.

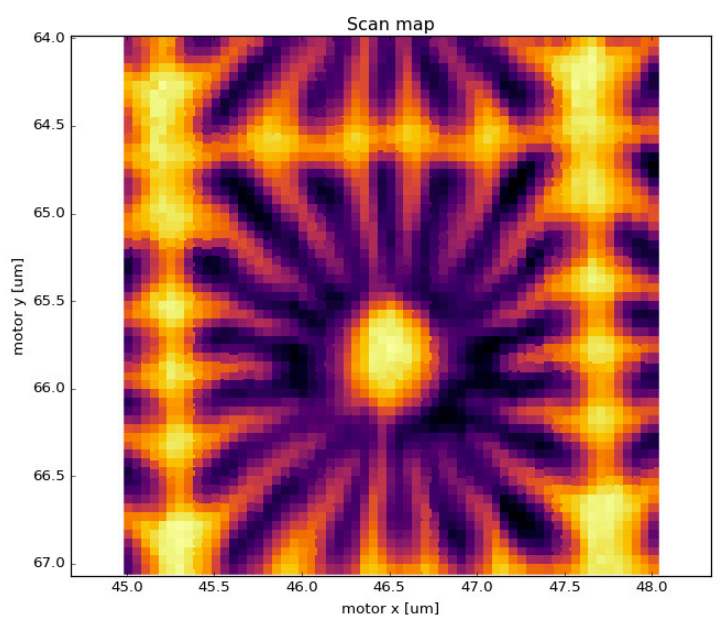

Figure. 2. XRF map of test structure made of Tungsten on Diamond substrate. Yellow is high intensity of $\mathrm{W} \mathrm{L}_{\beta}$ emission.
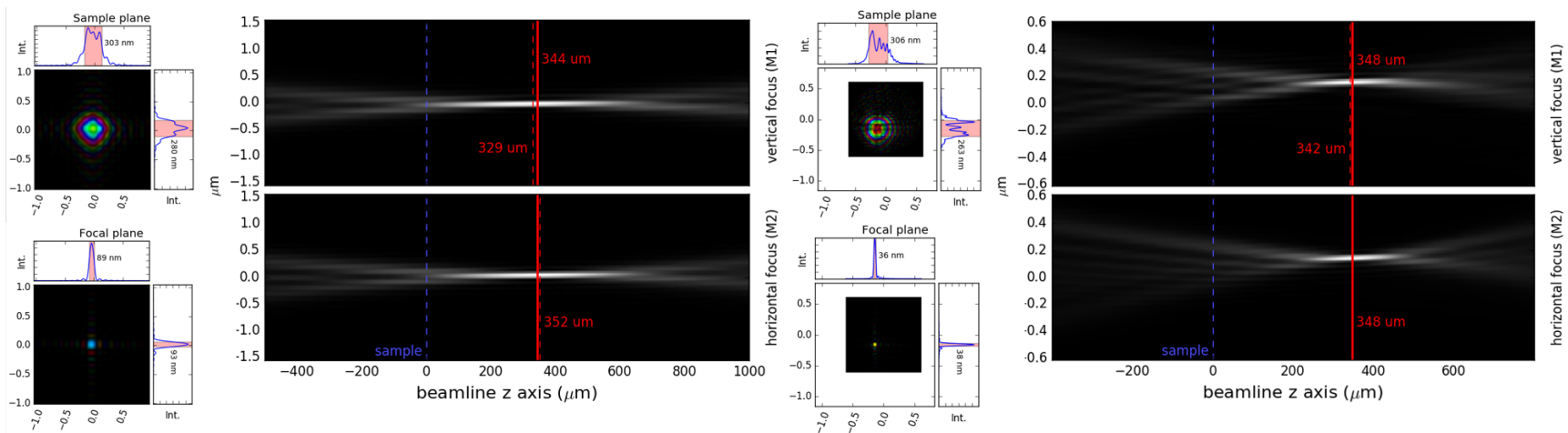

Figure. 3. Beam profile of the $\mathrm{KB}$ focus at $9.5 \mathrm{keV}$ (left pane) and $24.2 \mathrm{keV}$ (right pane) shown from ptychographic measurements and reconstruction of the probe. The measurement was done with the test structure in Figure 2. The resulting probe sizes are $89 \times 93 \mathrm{~nm}^{2}$ and $35 \times 39 \mathrm{~nm}^{2}$ respectively with differences due to wavelength dependent diffraction limit. 